\title{
Electroacupuncture for treatment of cervicogenic headache: A meta-analysis
}

\author{
Binbin Wu MD, PhD*, Gonghao Zhan MD, Jing Lin MD, Qingquan Lian MD, PhD* \\ Department of Anesthesiology and Pain Medicine, the Second Affiliated Hospital and Yuying Children's Hospi- \\ tal of Wenzhou Medical University
}

\begin{abstract}
Objective: Cervicogenic headache is a common aliment affecting clinically, but many current treatments failed to aid in long-term pain relief for these patients. Pharmacological agents such as analgesics and steroid base injections are among a few of current treatments utilized in clinical, however, their efficacy remains controversial, which the use of non-pharmacological based methods can be utilized as a treatment option. Therefore, we investigated the curative effect of electroacupuncture in treating cervicogenic headache using meta-analysis from 21 studies, and 6 studies were selected as the final articles.
\end{abstract}

Methods: We searched MedLine, Ovid, Embase, and Cochrane Library electronic databases, Wanfang and Weipu Chinese electronic databases, and the Chinese Biomedical Retrieval System using the key words of "electroacupuncture", "acupuncture" and "cervicogenic headache" for randomized or quasi-randomized controlled trials or controlled clinical trials.

Results: Six studies including 609 patients met the inclusion criteria, and these studies compared curative effect of electroacupuncture with other methods. Two trials reported greater overall effectiveness rate of electroacupuncture than the oral medicine subgroup (OR 8.16, 95\% CI 3.43-19.41; $P<0.00001$ ), but none of the others suggested the superiority of electroacupuncture versus anyother subgroup by a random model. A meta-analysis of all trials compared electroacupuncture with other treatments did not show significant difference (OR 2.17, 95\% CI 0.74 to 6.36; $P=0.16$ ) with a random model. No obvious publication bias concerning the effectiveness rate was identified with Begg's test.
Conclusions: Electroacupuncture shows higher effectiveness rate for cervicogenic headache treatment compared to oral treatment adopted in the included trials, but no advantage in overall effectiveness rate was observed, thus, it appears that electroacupuncture may not be a superior treatment for cervicogenic headache. However, the quality of the trials included was insufficient to reach any firm conclusion regarding the curative effect of electroacupuncture, so higher quality studies are required in the future.

Key Words: cervicogenic headache, electroacupuncture, effectiveness rate, meta-analysis.

\section{Introduction}

Headache is a common symptom associated with a high morbidity rate, it can be divided into many categories, for example, primary and secondary headaches, but its etiology has not been investigated extensively. Cervicogenic headache (CHE) is a chronic headache syndrome, caused by pathological changes in the bones and/or neck soft tissues, and always presents as pain and tenderness in the neck, resulting in stiffness and limitations of activity [1]. The pain could be aggravated as the disease progressing, and quality of life is seriously affected; nevertheless, the etiology and pathophysiology have been debated intensively. Besides, autoimmune inflammation, vascular ischemia, injury and inappropriate massage also can affect neurological function and lead to $\mathrm{CHE}[2,3]$.According to the theory of "stagnation of Qi and Blood may bring about pain" in traditional Chinese medicine (TCM), Qi refers to the vital substances that comprise the human body and the physiological functioning of the bowels, channels, viscera and collaterals, preserving life activities and reflect the resistance of the 
human body. The initial cause of the pain is inadequate of Qi, followed by blood block and results in stagnant veins and sluggish blood [5].

Acupuncturology is an important part of TCM that dates back thousands of years. Acupuncture can be conducted by inserting needles into specific points on the skin, the treatment is considered to regulate the channels and collaterals by harmonizing the Blood and Qi to improve immunity and promote the excitability and regeneration of nerves; the formation of collateral branches, blood circulation and muscle contraction can also be advanced, in turn, accelerating metabolism and the recovery of bodily functions. Recently, some studies reported acupuncture was an effective therapy for $\mathrm{CHE}$, and electroacupuncture is a therapy that combines the curative effects of traditional acupuncture and transcutaneous electrical nerve stimulation. Once the needles are inserted, low electrical current was propagated deep to the tissue, and the applications and results have been reproduced in many clinical and basic science research settings. Electroacupuncture provides advantages such as more accurate stimulus parameters and less manual intervention, and can be more effective to treat musculoskeletal pain compared to traditional manual acupuncture.

\section{Materials and Methods}

\section{Studies and Participants}

All randomized and quasi-randomized controlled trials of acupuncture for CHE treatment were searched, either in English or Chinese langauges. A total of 21 potentially relevant studies were included of those, 15 studies were excluded because of inadequate information. After the authors reviewed the quality of these remaining studies, a total of 6 trials with 609 participants were included in the final analysis.

\section{Types of intervention}

All studies utilized electroacupuncture in the experimental group, and any other conventional interventions as a control group. The studies utilized a variety of treatments, including oral medications (e.g., celecoxib and/or sodium dichlorophenolate), lidocaine injection, triamcinolone and vitamin B12, tuina. Acupuncture techniques included a variety of acupuncture points, Fengchi, Jingjiaji, Tianzhu, Baihui, and Ashi were involved in all or partly of these selected studies to relieve the associated pain. Fengchi has been primarily used to treat headache, neck pain, dizziness and help improve stroke sequela, and also is the most effective in relaxing neck muscles, facilitating the associated analgesia and was adopted in all the included trials. Jingjiaji was regarded as another curative acupuncture point, most known for improving cardiovascular function and regulating autonomic function, which might be related to the prognosis of CHE, therefore a valid treatment $[2,3,6,7]$. Moreover, stimulation at Tianzhu was a potent treatment for stiffness and aches in shoulder muscles, and it was used in the studies by Wang, Jia, Zhou, and $\mathrm{Hu}[2,6,7,8]$. Shuaigu was the joint of the Zutaiyang and Shaoyang, covered by the temporalis anatomically and was reported effective in treating headache, dizziness, trigeminal neuralgia and facioplegia, adopted in 4 trials $[2,3,7,8]$. Baihui was included in the studies by Wang, $\mathrm{Hu}$, Jia, and Qiu [2,5,6,8], and Ashi was used in those by Yang, Jia, and Qiu $[3,6,9]$.

\section{Outcome measures}

The primary outcome was patient recovery rates and symptom relief. The overall satisfaction was evaluated with clinical criteria at the end of treatment or each treatment course. To be noted the treatment duration varied amongst the studies,

\section{Search methods}

The MedLine, Ovid, Embase, and Cochrane Library electronic databases, and Chinese electronic databases, such as Wanfang and Weipu and the Chinese Biomedical Retrieval System were searched with the terms of "electroacupuncture", "acupuncture" and "cervicogenic headache" for randomized, quasi-randomized controlled trials and controlled clinical trials, published in English or Chinese. All randomized and quasi-randomized controlled clinical trials of electroacupuncture for the treatment of CHE were included, and all articles were reviewed manually by the authors to meet the minimum inclusion criteria,

\section{Selection of studies and methodological quality assessment}

Two review authors were responsible for scrutinizing the titles and abstracts from the searches, and the full 
texts of all potentially relevant studies were obtained for independent assessment. Two review authors determined the final selection of studies and any disagreements were resolved by consensus. The assessment of methodological quality accounted for differences at baseline between the treated and control groups. The blinding and overall completeness of patients were also evaluated.

\section{Data extraction and management}

Data on the patients, methods, intervention outcomes and results were obtained by the 2 review authors independently by using a data extraction form. In each included trial, electroacupuncture was compared with another treatment method alone, and any combined therapy data were excluded.

\section{Statistical analysis}

Statistical analysis were performed the using RevMan 5.0 software, provided by the Cochrane Collaboration, and $P<0.05$ was considered significant. Due to the clinical heterogeneity among the studies, the analysis was performed by using a random effect model for more conservative estimates. Dichotomous data are presented as odds ratios (ORs) with $95 \%$ confidence intervals (CIs). We checked all results for clinical and statistical heterogeneity, and $\mathrm{I}^{2}$ statistics were used for the evaluation of statistical heterogeneity $\left(\mathrm{I}^{2}\right.$ values of $50 \%$ or more indicate heterogeneity). $\mathrm{I}^{2}$ values of $25 \%$ or less were considered to indicate low and values of $75 \%$ or more to indicate high heterogeneity. Forest plots were used to present the meta-analysis, the vertical line representing the equivalence line between the methods was compared, and individual trials displayed horizontally with the squares were estimated for each trial. The area of the square was proportional to the amount of information, the horizontal line indicat- ed the $95 \% \mathrm{CI}$ and diamonds represented summary measures with the width corresponding to the $95 \% \mathrm{CI}$. Begg's funnel plot was used to assess publication bias.

\section{Results}

Twenty one potential studies were narrowed down to 6 studies due to inadequate information. The final 6 trials involved 609 participants were selected in this meta-analysis. Electroacupuncture was compared to local lidocaine injection in 1 trial [2], tuina and a modified electroacupuncture technique combined with tuina comprised 2 separate trials [3,7]. Electroacunpcture was also compared with pharmacological agents such as celecoxib and sodium dichlorophenolate in 1 trial [6], and with conventional acupuncture in 1 trial [9]. The clinical diagnostic criteria adopted in the trials conducted by Wang, Hu and Yang was initially described by Sjaastad [10]. The diagnostic criteria in the trial by Zhou only included neuropathic pain in the occipitalia and calvaria, the face was not involved [8]. The pain was almost always reported to radiate unilaterally, some patients complained of orbital pain, dizziness, and nausea but no clear neurological origin could be determined. Many of the patients reported worsen pain when fatigued or with holding certain head movements, such as a head bow for a long time. Several relevant tender points approximate presented as suitable candidates for accunpcture sites to improve vascular circulation and promote nerve relaxation. In this study, they excluded patients with a history of any cerebrovascular disease or cerebral tumors. The inclusion criteria in the study by Qiu strictly included CHE patients with known etiologies: cervical bone hyperplasia, injury to the muscle, ligament or joint capsule; or secondary to aseptic inflammation and conglutination, stimulating or compressed the neck nerves [9]. The patients presented bilateral occipital pain that radiated to the parietal region, accompanied

Table 1. The baseline characteristics of the 6 trials

\begin{tabular}{|c|c|c|c|c|c|c|c|c|c|c|c|}
\hline \multirow{2}{*}{ Trials } & \multirow{2}{*}{ Intervention } & \multicolumn{2}{|c|}{ No. of patients } & \multicolumn{2}{|c|}{ Age $($ Mean $\pm S D)$ y } & \multicolumn{2}{|c|}{ Gender(W/M) } & \multicolumn{2}{|c|}{ efficacy/inefficacy } & \multirow{2}{*}{ Follow up } & \multirow{2}{*}{ Jadad score } \\
\hline & & EA & Others & EA & Others & EA & Others & EA & Others & & \\
\hline Wang 2010 & EA VS Localized drug injection & 28 & 28 & $41.6 \pm 13.5$ & $42.5 \pm 12.6$ & $17(11)$ & $16(12)$ & $23 / 5$ & $26 / 2$ & NA & 3 \\
\hline Hu 2005 & EA VS Tuina & 26 & 28 & $47.50 \pm 12.95$ & $46.21 \pm 14.12$ & 13(13) & $15(13)$ & $24 / 2$ & $26 / 2$ & $12 \mathrm{~m}$ & 2 \\
\hline Yang 2004 & EA VS Tuina & 50 & 50 & $25-67$ & $28-56$ & $33(17)$ & $38(12)$ & $45 / 5$ & $44 / 6$ & $12 \mathrm{~m}$ & 2 \\
\hline Jia 2007 & EA VS Oral medicine & 40 & 40 & $\mathrm{NA}$ & NA & Total & $44(36)$ & $39 / 1$ & $30 / 10$ & $\mathrm{NA}$ & 2 \\
\hline Zhou 2008 & EA VS Oral medicine & 63 & 54 & $36(19-63)$ & $34(23-65)^{\#}$ & $29(34)$ & $26(28)$ & $56 / 7$ & $28 / 26$ & $2 \mathrm{~m}$ & 1 \\
\hline Qiu 2008 & EA VS CA & 31 & 30 & $41.23 \pm 7.06$ & $40.56 \pm 6.27$ & $18(13)$ & $14(16)$ & $29 / 2$ & $26 / 4$ & NA & 3 \\
\hline
\end{tabular}

\#median(range); EA: electroacupuncture; CA: conventional acupuncture; y: year; m: month 


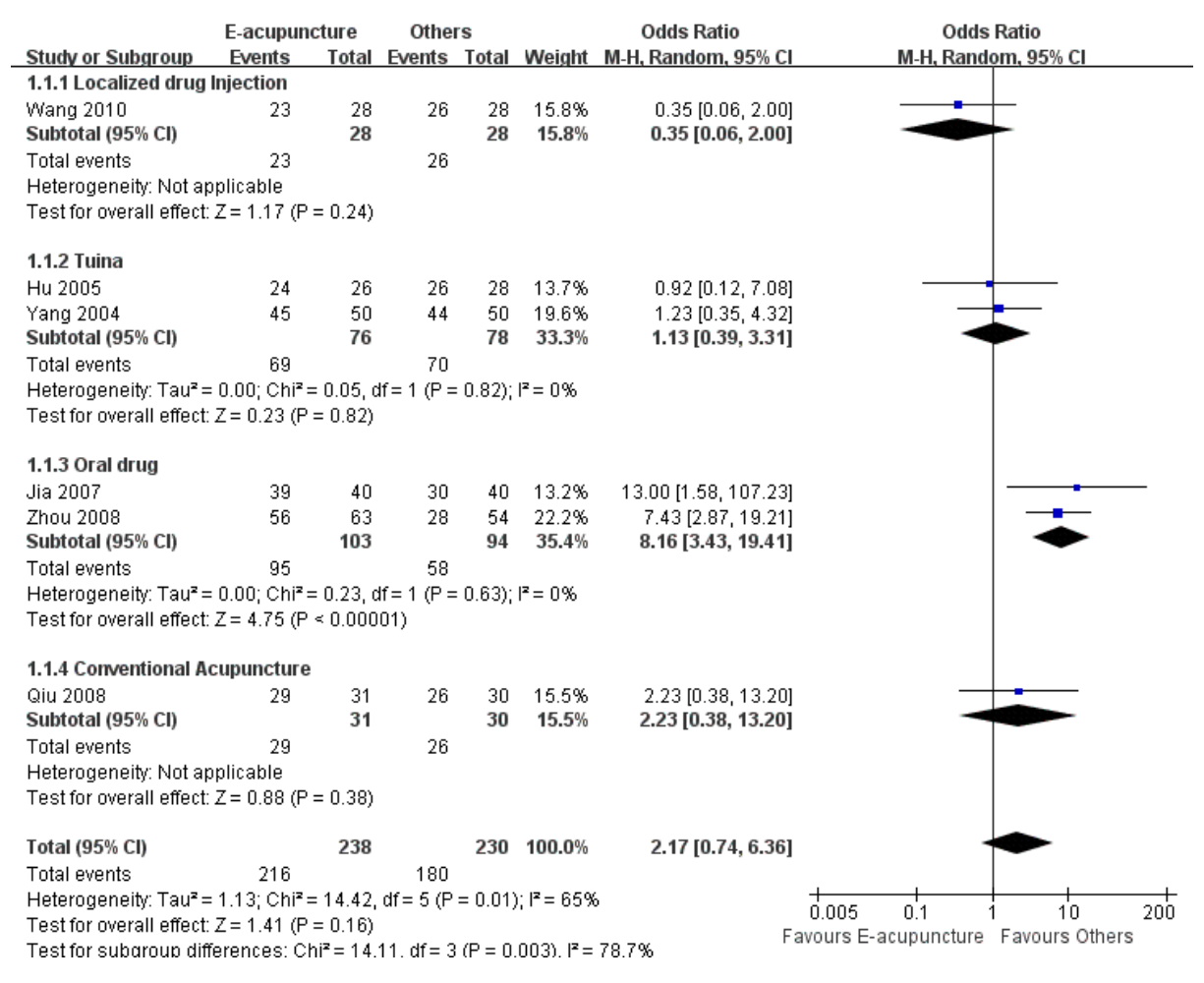

Figure 1. Meta-analysis of the effectiveness rates of electroacupuncture and other interventions.

by swelling and tension. Other causes of headache and intracranial organic diseases were ruled out by $\mathrm{CT}$ and MRI. However, the diagnostic criteria in Jia's study was not described, and some other studies did not provide the basic information of all participants, such as age, disease course, and medical history of diabetes, hypertension and pathological changes in bones [6]. Table 1 shows the baseline characteristics of the populations of the studies.

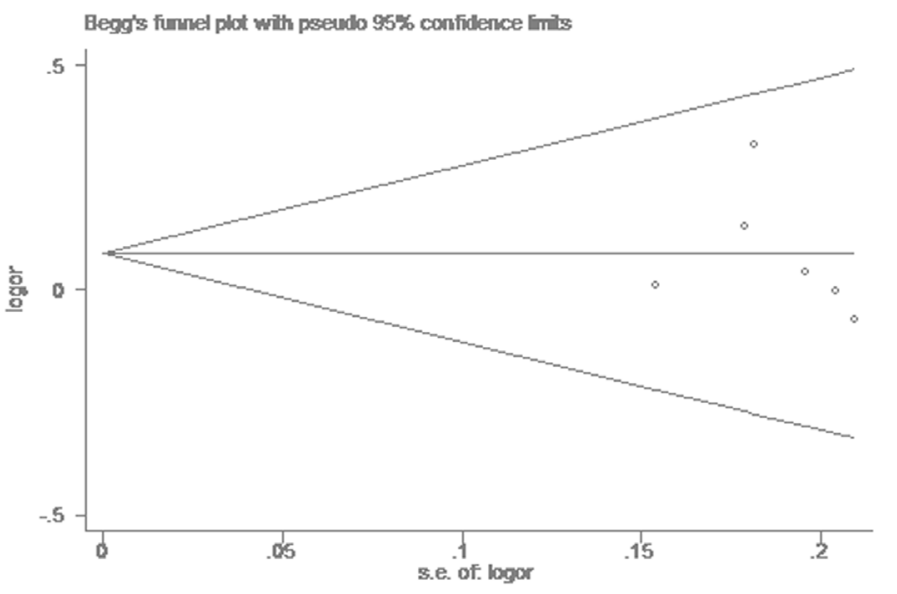

Figure 2. Begg's test of publication bias in the effectiveness rate of electroacupuncture versus other interventions.
The rates of effectiveness were reported in all included studies, the overall effective rates in the electroacupuncture group and the compared subgroups were $90.76 \%$ and $78.26 \%$, respectively. The trials by Jia and Zhou reported significantly greater overall effectiveness rates for electroacupuncture than oral medication (OR 8.16, 95\% CI 3.4319.41; $P<0.00001 ; \mathrm{I}^{2}$ $=0 \%$ ). With the exception of this finding, none of the remaining analysis suggested the superiority of electroacupuncture over any other treatment, including local drug injection (OR 0.35, 95\% CI 0.06-2.00; $P$ $=0.24$, the test for heterogeneity was not applicable), tuina (OR 1.13, 95\% CI 0.39-3.31; $P=0.82, \mathrm{I}^{2}=0 \%$ ), and conventional acupuncture (OR 2.23, 95\% CI 0.38$13.20 ; P=0.38$, the test for heterogeneity was not applicable). The meta-analysis of all trials comparing electroacupuncture with other treatments did not indicate a significant difference (OR 2.17, 95\% CI 0.74 to $6.36 ; P=0.16$ ) with a random model (Figure 1). The possibility of publication bias was assessed by Begg's test, and no obvious publication bias in the effectiveness rate (Figure 2, Begg's test: $P>0.728$, continuity corrected).

\section{Discussions}

Cervical headache seriously affects people's health 
and quality life all over the world, approximately $47 \%$ of the global population suffers from headaches $[11,12]$, and $15-20 \%$ of those headaches are cervicogenic. Compared with males, females seem more predisposed to cervicogenic headaches, and the morbidity in females is about four times as many as males, especially those during 20-60 years old. Some epidemiological researches suggested a higher prevalence of headache in adults with neck pain $[13,14]$. In China, the incidence of CHE was reported to be 25 per 1000 people, accounting for $14-18 \%$ of headaches in the population [15].

Many hypotheses regarding cervicogenic pathophysiology were proposed, one example was persistenting physical labour could result in sustained muscle contraction, and finally caused chronic strain of the muscles, ligaments and muscular fascia. Overtime, the strain directly stimulated neural stems and/or nerve endings that passed through soft tissues, ultimately resulted in nociceptive input and pain [14]. Degenerative changes in the cervical vertebra and intervertebral discs might also be the causes of foraminal stenosis. Thus, pain could be caused by stimulation or compression of the nerves or blood vessels that passed through the intervertebral foramen and led to inflammation. Besides, the degeneration of cervical intervertebral discs was always accompanied by aseptic inflammation and edema, and the release of inflammatory mediators peripherally resulted in pain [16]. Additionally, cumulative strain on the nuchal ligamen, longterm mental and emotional stress might also be central causes of cervical headaches.

Various therapies have been used to treat CHE, including pharmacological agents, manual physical interventions, transcutaneous electrical nerve stimulation therapy, local injections of anesthetics, and invasive surgical therapies. Unfortunately, the mechanisms regarding $\mathrm{CHE}$ etiology remains unclear. TCM, as a complement to conventional medicine, has become popular worldwide and in recent decades [17]. In China, acupuncture has been used as a viable treatment for various health problems for over 5,000 years, such as neurological diseases, neuropathic pain and psychological disorders. Although the mechanism of acupuncture analgesia remains controversial and lacking objective evidence, many studies theorized the central nervous system and pain inhibitory systems could be modulated by acupuncture; and peripheral afferent pathways transmitting acupuncture impulses were activated following stimulation, and these impulses propagated to the hypothalamus-pituitary system via the spinal cord anterolateral tract. Subsequently, the changes in the blood and cerebrospinal fluid showed higher levels of beta-endorphins, indicating an increased in activity of the hypothalamus-pituitary circuit and resulted in greater release of endogenous analgesic products. Furthermore, opioid receptor motifs and tone (mu, delta, and kappa receptors) in the spinal cord of the rats also might play important roles in sustained pain relief $[18,19]$.

In this systematic review, we focused on a comparison of the efficacy of electroacupuncture to local drug injection (lidocaine), tuina, oral drugs (e.g., celecoxib and/or sodium dichlorophenolate), and conventional acupuncture. A total of 6 trials with a total of 609 participants were included. The results showed that electroacupuncture was more effective than oral drug administration (celecoxib and sodium dichlorophenolate), but not obviously superior to other interventions for CHE treatment. Despite electroacupuncture did not show any advantage compared with the other treatments mentioned above by a random model, the difference was significant when a fixed-effects model was used (data not shown). The advantages of electroacupuncture should be evaluated further because of the limitations of the included studies. Due to some methodological shortcomings, specifically, the method of randomization was not reported for all the included trials, we cannot make any firm conclusion regarding superiority of electroacupuncture towards treating CHE. High-quality RCTs with internal validity are needed in the future to support the use of electroacupuncture as a viable treatment.

In conclusion, electroacupuncture might not be superior to analgesic medications for CHE treatment, although the tendency towards a higher effectiveness rate for electroacupuncture versus oral medicine treatment (e.g., celecoxib and/or sodium dichlorophenolate). However, the quality of the trials included was insufficient to reach any confirmed conclusion regarding the effectiveness of electroacupuncture treatment, and this should be addressed in larger and better quality studies in the future. 


\section{Acknowledgements}

The authors thank Taha Abdullah M.S., department of Physiology, Northwestern University Feinberg School of Medicine for language help.

Disclosure of Funding: The study was supported by Wenzhou Science and Technology Project (Y20160392) and the Foundation of Zhejiang Medical Association (2015ZYC-A28)

Conflict Interests Disclosure: The authors have no conflicting interests to disclose.

Corresponding Authors: Qingquan Lian, Binbin Wu. Department of Anesthesiology and Pain Medicine, the Second Affiliated Hospital and Yuying Children's Hospital of Wenzhou Medical University, NO. 109 Xueyuan Road, Wenzhou, Zhejiang, China. Binbin Wu:wbb19880117@163.com; Qingquan Lian: lianqingquanmz@163.com. Tel.:+86 0577-88002927

Editor: Jun-Ming Zhang,MD, MSc, Professor and Vice Chair for Research, Department of Anesthesiology, University of Cincinnati. jun-ming. zhang@uc.edu

\section{Additional publication details}

Journal short name: Transl Perioper \& Pain Med Received Date: Oct 28, 2016

Accepted Date: March 3, 2017

Published Date: March 4, 2017

Transl Perioper \& Pain Med 2017; 2(2):6-12

\section{Citation and Copyright}

Citation: Wu B, Zhan Z, Lin J, Lian Q. Electroacupuncture for treatment of cervicogenic headache: a meta-analysis. Transl Perioper \& Pain Med 2017; 2(2): 6-12

Copyright: (C) $2017 \mathrm{Wu} \mathrm{B}$, et al.This is an open-access article distributed under the terms of the Creative Commons Attribution License, which permits unrestricted use, distribution, and reproduction in any medium, provided the original author and source are credited.

\section{References}

1. Odonkor CA, Tang T, Taftian D, Chhatre A. Bilateral intra-articular radiofrequency ablation for cervicogenic headache. Case Rep Anesthesiol 2017; 2017:1483279. PMID: 28149652

2. Wang J. The injection of analgetic in $\mathrm{C} 2$ for the treatment of cervical headache (in Chinese). Medical Information 2010; 23:122.

3. Yang TH, Zhao KZ, Zhou QY. The effect observation of the therapy of electroacupuncture combined with tuina for cervical headache (in Chinese). Journal of External Therapy of TCM 2004; 13:34.

4. Assapun J, Uthaikhup S. Localized pain hypersensitivity in older women with cervicogenic headache: A quantitative sensory testing study. J Oral Facial Pain Headache 2017; 31:80-6. PMID: 28228424

5. He L, Zhou D, Wu B, Li N, Zhou MK. Acupuncture for Bell's Palsy. Cochrane Database Syst Rev 2004; (1): CD002914. PMID: 14973996

6. Jia YB. Electroacupuncture for treating 40 cases of cervical headache (in Chinese). Shanghai J Acu-Mox 2008; 27:8.

7. Hu WQ, Xu SW. Electroacupuncture combined with tuina for the treatment of cervical headache (in Chinese). J Chin Integr Med 2005; 3: 310-11.

8. Zhou XM, Sun H, Chen J. The electroacupuncture in points in occipital region for the therapy of cervical headache (in Chinese). Shanghai J Acu-Mox 2008; 27:30.

9. Qiu XE. The treatment for 31 cases of cervical headache with electroacupuncture (in Chinese). Journal of Traditional Chinese Medicine 2008; 49:1099100 .

10. Sjaastad O, Fredriksen T, Pfaffenrath V. Cervicogenic head ache: diagnostic criteria. The Cervicogenic Headache International Study Group. Headache 1998; 38: 442-5. PMID: 9664748

11. Stovner Li, Hagen K, Jensen R, Katsarava Z, Lipton R, et al. The global burden of headache: a documentation of headache prevalence and disability worldwide. Cephalalgia 2007; 27: 193-210. PMID: 
12. Jensen R, Stovner L. Epidemiology and comorbidity of headache. Lancet Neurol 2008; 7: 354-61.

PMID: 18339350

13. Haldeman S, Dagenais S. Cervicogenic headaches: a critical review. Spine J 2001; 1: 31-46. PMID: 14588366

14. Page P. Cervicogenic headaches: an evidence: led approach to clinical management. The Int J Sports Phys Ther 2011; 6: 254-66. PMID: 22034615

15. Zhu SR, Zhu SY, Hou KD. The diagnosis and therapy for the cervicogenic headache (in Chinese). Chinese Journal of Pain Medicine 2005; 11: 330.

16. Uthaikhup S, Sterling M, Jull G. Cervical musculoskeletal impairment is common in elders with headache. Man Ther. 2009; 14: 636-41. PMID:19213591

17. Zijlstra FJ, van den Berg-de Lange I, Huygen F, Klein J. Anti-inflammatory actions of acupuncture. Mediators of Inflamm 2003; 12: 59-69. PMID: 12775355

18. Chen XH, Han JS. Analgesia induced by electroacupuncture of different frequencies is mediated by different types of opioid receptors: another cross-tolerance study. Behav Brain Res 1992 ; 47:143-49.

PMID:1350448

19. Saha JK, Xia J, Engle SK, Chen YF, Glaesner W, et al. A model of controlled acute hyperglycemia in rats: Effects of insulin and glucagon-like peptide-1 analog. J Pharmacol Exp Ther 2006; 316:1159-64.

PMID: 16280413 